\title{
Controle de potência de transmissão em VANETs: Uma abordagem utilizando Teoria dos Jogos
}

\author{
Thiago Montenegro Uchôa ${ }^{1}$, Heitor S. Ramos ${ }^{2}$, Stênio Flávio de Lacerda \\ Fernandes ${ }^{1}$ \\ ${ }^{1}$ Centro de Informática - Universidade Federal de Pernambuco (UFPE) - Recife - PE - \\ Brazil. \\ ${ }^{2}$ Instituto de Computação - Universidade Federal de Alagoas (UFAL) - Maceió - AL - \\ Brazil. \\ tmu@cin.ufpe.br, heitor@ic.ufal.br, sflfecin.ufpe.br
}

\begin{abstract}
This work proposes a power control algorithm to reduce the impact of high power transmission in Vehicular Ad Hoc Networks (VANETs). Based on game theory, we propose GRaPhic, a technique that encourages the devices connected to a VANET to reduce their transmission power. This algorithm provides enough incentive for a network does not deliberately increase its transmission power. In GRaPhic, we propose a utility function capable of reducing the transmission power of vehicles.
\end{abstract}

Resumo. Este trabalho propõe um algoritmo de controle de potência para reduzir o impacto de altas potências de transmissão em VANETs. Baseado em Teoria dos Jogos, propomos GRaPhic, uma técnica que estimula os dispositivos conectados à VANET a reduzir suas potências de transmissão. Este algoritmo provê incentivo suficiente para esta rede não aumentar deliberadamente suas potências de transmissão. Para isto, é proposta uma função utilidade capaz de reduzir a potência de transmissão de veículos, se um conjunto de condições for satisfeito.

\section{Introdução}

A comunicação em VANETS se dá entre veículos V2V (Vehicle-to-Vehicle) ou veículos e infraestrutura V2I (Vehicle-to-Infraestructure). Ambas as formas de comunicação têm atraído o interesse de pesquisas, principalmente devido à mobilidade que se tem nestas redes (WANG; FAN; LETAIEF, 2012). Em contrapartida, a mobilidade aliada à alta concentração de transmissores, instalados nos veículos, pode gerar um problema relacionado à interferência entre os nós da rede. Em um ambiente de grande concentração veicular as taxas de transmissão podem ser reduzidas devido à alta interferência entre os nós da rede. Para reduzir este efeito, a abordagem escolhida foi a redução de potência de transmissão em cenários de grande concentração veicular. Para modelar o algoritmo de redução de potência de transmissão, foi escolhida a teoria dos jogos, como descrita abaixo.

A natureza da escolha da potência de transmissão em nós de uma rede pode ser modelada matematicamente com a Teoria dos Jogos não cooperativos. Isto se dá porque, neste caso específico, os nós da rede tentarão obter o maior payoff possível, ou seja, a melhor relação SINR (Signal to Interference plus Noise Ratio). Porém, com o uso de teoria dos jogos não cooperativos, as escolhas são feitas sem levar em 
consideração os efeitos do SINR nos outros nós. Ou seja, as escolhas dos nós serão baseadas somente na maximização de seu SINR, independentemente se isto irá piorar, ou não, o SINR dos nós vizinhos.

Em contrapartida, a teoria dos jogos cooperativos é uma ferramenta poderosa na análise de comportamento de jogadores, como o exemplo de nós de uma rede, garantindo que os mesmos não tenham incentivos a desviar unilateralmente nos seus comportamentos. A teoria dos jogos cooperativos é um importante arcabouço no desenvolvimento de mecanismos descentralizados, nos quais o sistema é capaz de se auto organizar de maneira mutuamente satisfatória (CHEN, 2014). O diferencial deste trabalho é o de propor um algoritmo, o GRaPhic, que modela matematicamente a escolha da potência de transmissão utilizando teoria dos jogos cooperativos. O objetivo é aumentar a relação de sinal ruído interferência (SINR), modelando matematicamente o comportamento de veículos que trocam informações do tráfego veicular em um ambiente de grande concentração veicular, estimulando a redução da potência de transmissão dos módulos de comunicação.

As demais seções estão organizadas da seguinte forma: A Seção 2 introduz os principais trabalhos que envolvem telecomunicações utilizando teoria dos jogos. A Seção 3 detalha a metodologia proposta por este trabalho. A Seção 4 mostra os principais resultados obtidos com as simulações. A Seção 5 conclui o trabalho e apresenta os trabalhos futuros.

\section{Trabalhos Relacionados}

As principais abordagens envolvendo teoria dos jogos são subdivididas em teoria dos jogos: não cooperativo e cooperativo. A primeira se utiliza quando se quer modelar cenários onde os jogadores tomam decisões de forma a não se preocupar com os efeitos nos outros jogadores. Na teoria dos jogos não cooperativos o objetivo de cada jogador é maximizar sua função utilidade sem levar em consideração os efeitos de suas jogadas no payoff dos outros jogadores. No caso específico do cenário de comunicação, será mostrado que, apesar de largamente utilizada, a teoria dos jogos não cooperativos leva, muitas vezes, a resultados de uso da máxima potência de transmissão. Dependendo do cenário estudado, este resultado pode ou não ser prejudicial.

Por outro lado, quando utilizado teoria dos jogos cooperativos, é estimulado que os jogadores não tenham incentivos a desviar unilateralmente. Para isto, duas metodologias são utilizadas: (i) baseada em reputação, (ii) em preço. A primeira metodologia tem por filosofia isolar os nós que não cooperam. Em um cenário de comunicação, os nós que retransmitem mais informações, são classificados como cooperadores e seus pacotes são repassados por outros nós. Já no caso de nós que não cooperam, seus pacotes não são repassados. Para a metodologia baseada em preço, cada nó da rede tem associado a ele um preço pago para repassar pacotes de outros nós e o preço pago para toda a rede por repassar seus pacotes. Quando repassar pacotes de outros nós, ganha créditos para quando for necessário passar seus pacotes.

O controle de potência de transmissão se mostra eficiente na redução da interferência e aumento da capacidade na transmissão de dados em redes sem fío (DOUROS; POLYZOS, 2011). Neste trabalho, são mostrados exemplos de aplicação do controle de potência de transmissão. Apesar do foco desta seção ser a revisão de 
trabalhos realizados em VANETs, muitos resultados referentes às MANETs e redes celulares são mostrados.

\subsection{Aplicações de Teoria dos Jogos Não Cooperativos}

Femtocell (FUJITSU, 2013) é um conceito de células de comunicação utilizando estações base (BS). Elas tipicamente são diferentes das BS tradicionais pelo seu reduzido alcance, 10m em média. Em (HAIDER et al., 2015) é proposto o uso de Mobile Femtocell (MFemtocell) para VANETs como trens, ônibus, táxis ou veículos particulares. Um dos maiores desafios para a aplicação das femtocells e mfemtocells está na interferência mútua entre as mesmas. Com o intuito de resolver o problema da interferência entre células, (HA; LE, 2014) propõe algoritmos de controle de potência distribuído e associação de distribuição de BS que possam manter a SINR em um nível determinado como bom. Um usuário terá a sua disposição células de vários tipos como: macrocélulas, microcélulas, picocélulas e femtocélulas. O algoritmo proposto ajuda na escolha de qual célula se conectar, baseado na qualidade do canal de comunicação.

O cenário de múltiplos nós de origem e destino, em uma rede sem fio, foi modelado utilizando teoria dos jogos não cooperativos repetitivos. Uma abordagem que visa otimizar a taxa de entrega de pacotes e SINR. Em (SMITH et al., 2014) são utilizados jogos com informações incompletas para implementações baseadas em IEEE 802.11 .

Em (HAN; LIU, 2005) é proposto um algoritmo de controle de potência de transmissão e throughput não cooperativo para comunicações sem fio. A metodologia proposta é centralizada, possui limitações de valores de SINR e visa maximizar a taxa de transmissão dos veículos que estão na rede. Algumas limitações foram notadas, como a falta de detalhamento sobre as simulações, assim como o detalhamento a respeito dos algoritmos propostos.

\subsection{Aplicações de Teoria dos Jogos Cooperativos}

Em (KHAYATIAN; SAADAT; ABOUEI, 2013) foi proposto um método que utiliza a teoria dos jogos no controle da potência de transmissão associado ao roteamento cooperativo das informações. Deixa-se de trabalhar isoladamente com roteamento, sem a análise da camada física. Os nós são divididos em três categorias: origem, relays e destino. Os últimos são considerados as estações rádio base. $\mathrm{O}$ sistema de controle cooperativo é do tipo descentralizado. A transmissão da origem ao destino é feita utilizando dois slots. O primeiro é um broadcast da origem para todos os nós em sua área de alcance. O segundo é a transmissão do nó origem e relay para as BS (Base Station). A comunicação é feita em três etapas: 1) formação da coalizão, 2) alocação cooperativa de potência e 3) transmissão de dados.

Em (TINGTING CHEN, 2011) os autores estudaram uma forma de estimular que os nós em uma VANET cooperem, transmitindo os pacotes para o destinatário, obedecendo à sua tabela de roteamento. Os principais mecanismos de recompensa para roteamento sem fio são: 1) Sistemas de reputação; e 2) Sistema de crédito. É argumentado que o primeiro sistema de recompensa não é adequado para aplicações de VANETs por causa da dinâmica das VANETs. Como nas VANETs a arquitetura de rede é bastante dinâmica, é argumentado que o sistema de crédito é mais eficiente em termos de resultados que o sistema de reputação. Neste estudo só existe um protocolo de roteamento. Não são estudados cenários com mais de um protocolo de roteamento 
visando a simplificação do cenário. A ideia é modelar a transferência de mensagens da origem ao destino como um jogo de coalizão de encaminhamento de informações. Para isto, são definidas funções de payoff para cada um dos nós integrantes da coalizão. Estas funções são projetadas levando em consideração a formação de um jogo que seja cooperativo e estável, estando este jogo modelado com teoria dos jogos utilizando a definição de core.

Em outros estudos como (YUYI LI, 2012), os autores desenvolveram mecanismos que permitem a seleção de gateway e formação da coalizão. Este trabalho, em específico, foca em teoria dos jogos cooperativos. O critério para escolha dos gateways é a máxima taxa de transmissão que o candidato pode receber da BS (estação base). Para a formação da coalizão, a função utilidade foi desenvolvida visando o incentivo de outros veículos a estabelecer conexões extras com outros nós. A função utilidade é variada baseada no número de nós de comunicação. Neste trabalho foi considerada uma área de $4 \mathrm{~km} X 4 \mathrm{~km}$. Os veículos estão localizados de forma aleatória nesta área. Este estudo envolveu a análise tanto de V2V quanto de V2I. As principais limitações do trabalho são que os autores não utilizam padronização IEEE 802.11p, que é desenvolvida especificamente para o uso em VANETs e está amplamente utilizada em pesquisas no meio acadêmico.

O foco deste trabalho está no esforço em reduzir a interferência entre veículos concentrados em uma determinada área, urbana ou não, através da diminuição da potência de transmissão entre os veículos. As abordagens já utilizadas de controle de potência de transmissão normalmente não utilizam teoria dos jogos, mas outras ferramentas para modelar matematicamente o algoritmo de controle de potência de transmissão. Com a finalidade de reduzir a interferência entre os veículos, os cenários estudados utilizam jogos cooperativos de controle de potência de transmissão e potência de transmissão fixa. Estas duas abordagens são comparadas, o que é um diferencial em relação a outros trabalhos já desenvolvidos. Ao contrário dos trabalhos já desenvolvidos, onde as simulações não consideram cenários de concentração veicular, foram conduzidas simulações considerando cenários de concentrações veiculares variadas e com diferentes valores de potência de transmissão inicial. Assim, pretende-se analisar a influência da concentração veicular e potência de transmissão nos resultados do algoritmo proposto.

\section{GRaPhiC: Um algoritmo de controle de potência de transmissão utilizando Teoria dos Jogos}

\subsection{Algoritmo Cooperativo}

O cenário cooperativo consiste de veículos que se comunicam utilizando de teoria dos jogos cooperativos para decidir se devem diminuir ou não a potência de transmissão. O objetivo de cada nó é maximizar sua função utilidade, como pode ser observado na Figura 1. Porém o algoritmo induz os jogadores, transmissores veiculares, a decrementar sua potência de transmissão, considerando a potência do transmissor que enviou o pacote.

Antes de analisar a lógica do algoritmo, se faz necessário apresentar a sua função utilidade, $u_{i}$, representada em (1).

$$
u_{i}=A \ln (1+\operatorname{SINR})-B(\text { txPower })
$$


Para o cálculo da função utilidade acima, foi considerada a taxa máxima de transmissão de Shannon, primeiro termo da equação, e um fator decrementador em função da potência de transmissão do veículo. Se a função utilidade fosse composta apenas do primeiro termo de (1), com a maximização da função utilidade, nenhum nó iria tentar reduzir sua potência de transmissão. Tentaria apenas aumentar sua potência de transmissão, com o intuito de aumentar a SINR e, consequentemente a função utilidade. Para induzir um comportamento cooperativo, foi introduzida a segunda parcela do cálculo de $u_{i}$. Este segundo termo é o responsável por controlar a potência de transmissão do veículo. Os fatores que multiplicam o logaritmo natural e a taxa de transmissão ( $A$ e $B$ ) foram encontrados de forma empírica, onde $A=1$ e $B=0,009$. A escolha foi feita baseada nos resultados do SINR encontrados nas simulações, onde os SINRs se tornaram máximos para estes valores de fatores multiplicativos. Simulações foram realizadas variando os valores de $A$ e $B$. Para cada simulação realizada com variações de $A$ e $B$ foram analisados os resultados de SINR. Os melhores resultados de SINR foram os encontrados para os valores escolhidos de 1 e 0,009 , respectivamente.

Explicada a função utilidade, se faz necessária a explanação do algoritmo implementado no GRaPhiC. Alguns passos como calcular a função utilidade e algumas comparações da potência de transmissão e função utilidade são mostrados no diagrama abaixo. Como o objetivo é maximizar a função utilidade, o valor da função utilidade é sempre comparado com um valor máximo armazenado em memória. Se o valor atual for menor que o máximo armazenado, a potência de transmissão é reduzida para tentar aumentar o valor da função utilidade.

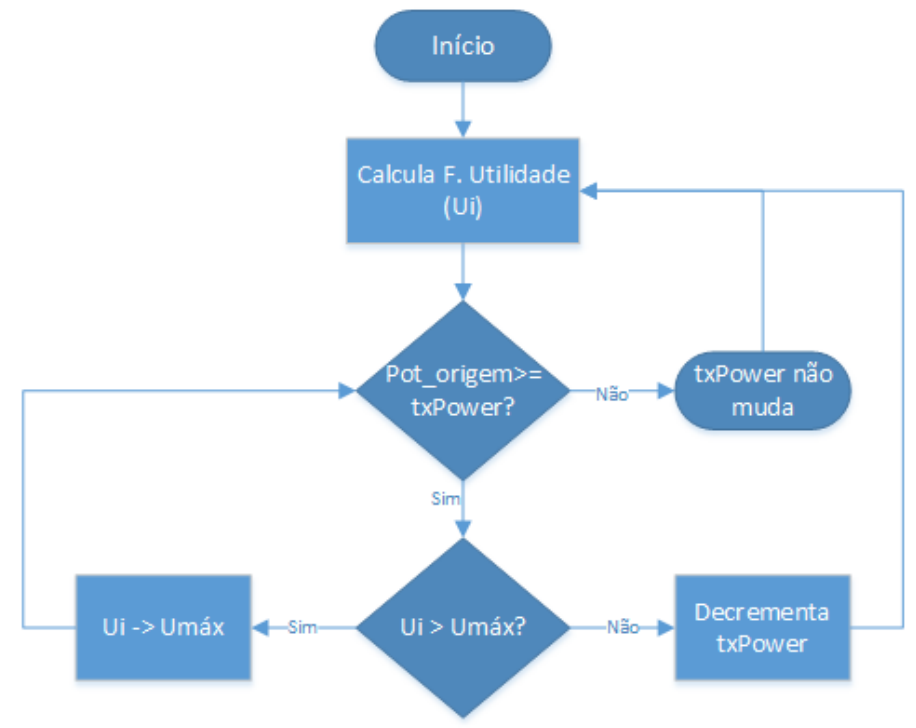

Figura 1: Algoritmo Cooperativo.

\subsection{Métricas do Sistema}

Para provar que o algoritmo proposto é efetivo, foram realizadas simulações levando em consideração condições realistas. Comparando com (LI et al., 2014), o sistema proposto é modelado e simulado inicialmente com áreas de $8 \times 8 \mathrm{~km}$ e $10 \times 10 \mathrm{~km}$. Para analisar cenários com diferentes concentrações de veículos, foram simuladas rotas com 250, 500, 1000 e 2000 veículos. Os veículos se comunicam através 
de broadcasts para todos os seus vizinhos em suas áreas de cobertura. Assim como em (YERRAMALLI; JAIN; URBASHI, 2014), a relação entre o número de veículos e a SINR média é analisada. As variáveis dependentes são: (i) potência de transmissão instantânea, (ii) SINR. Já as independentes são: (i) potência de transmissão inicial, (ii) número de veículos na simulação, (iii) área de interesse.

A influência das variáveis independentes sobre as variáveis dependentes é analisada. Espera-se que com o aumento na potência de transmissão, a SINR decresça, como pode ser observado na equação em (1). O aumento no número de nós na simulação deve aumentar a interferência na área de interesse, diminuindo a SINR e a taxa de transmissão. Todas estas análises devem ser mostradas com mais detalhes na seção de resultados das simulações.

\subsection{Métodos de Avaliação}

Para avaliar a metodologia proposta, GRaPhiC, se fez necessário simular os veículos gerando tráfego de dados na rede formada pelos transmissores veiculares. Para simular o tráfego de informações, foram gerados broadcasts quando os veículos se envolviam em acidentes. Todas as simulações foram realizadas utilizando o Veins. Nas simulações realizadas, os veículos que se envolvem nestes acidentes, enviam um broadcast para seus vizinhos (V2V) e Base Stations (V2I). Nas simulações as colisões são induzidas. Assim, nos instantes onde não há acidente, não há broadcast, a não ser aqueles de retransmissões de acidentes anteriores através de comunicação V2V. Antes de cada transmissão, quando um airframe é recebido, o algoritmo proposto é executado, analisando o ambiente para analisar a possibilidade de redução da potência de transmissão da unidade de bordo (OBU) do veículo em questão. $\mathrm{O}$ algoritmo proposto não incrementa a potência de transmissão, apenas reduz ou a mantém constante, desta forma foi possível analisar o cenário de uma forma simplificada.

O cenário de simulação estuda as comunicações tipo V2V e V2I. Para isto, são considerados grids com blocos de $100 \times 100 \mathrm{~m}^{2}$. Os últimos simulam uma área formada por quarteirões. Para avaliar o efeito da concentração veicular no GRaPhiC foram criados dois tipos de grid. O primeiro tipo tem 80 cruzamentos na horizontal e 80 na vertical. O segundo tem 100 cruzamentos na vertical e 100 na horizontal. A BS é simulada no centro do cenário e os veículos se locomovem pelo cenário.

Por outro lado, cada veículo tem que ter uma rota pré-selecionada. Cada rota tem no mínimo $100 \mathrm{~m}$ de distância. Todas as rotas são simuladas utilizando o randomTrips.py (SUMO, 2013). Este script permite que o número de veículos na área de interesse seja gerado de forma constante ou aleatória, seguindo uma distribuição binomial. Para avaliar o efeito da quantidade de veículos em uma determinada área, foram simulados cenários com até $2000,1000,500$ e 250 veículos. Para realizar as simulações, o cenário inicialmente é considerado vazio e novos veículos são inseridos na área de interesse a cada 0,5,1,2 e 4 segundos. Quando maior este intervalo, menor a quantidade de veículos no final da simulação, que se manteve constante para todos os cenários.

Para simular os acidentes, determinados nós são escolhidos e enviam broadcasts simulando uma mensagem de acidente. Nas simulações conduzidas, cada cenário tem 25 simulações de acidente. Em todos os cenários, os mesmos nós simulam acidentes. $\mathrm{O}$ número de veículos que simulam acidentes é proporcional ao número de veículos simulados. Com o número de veículos aumentando, também será maior o número de 
veículos simulando acidentes. Este número varia entre 24 e 199, dependendo do número de veículos na simulação.

Foram realizadas diversas simulações, levando em consideração variações das variáveis independentes, como mostrado na Figura 2. Para analisar as simulações realizadas, inicialmente foi simulado um cenário de grid sem obstáculos. Por fim, também foram simulados veículos com diferentes valores iniciais de potência de transmissão: 30, 50, 80 e 100mW. A escolha destes valores de potência de transmissão se basearam em trabalhos já realizados anteriormente e são especificados em equipamentos utilizados em simulações reais.

O objetivo das simulações é analisar a razão SINR para os diferentes cenários. Para obter significância estatística, todos os cenários foram simulados por 30 vezes cada, com coeficiente de confiança de 95\%. As simulações são realizadas, cada uma, simulando rotas veiculares distintas. Dependendo do cenário, várias simulações, como em (RAWAT; POPESCU; OLARIU, 2011), têm o tempo de simulação de 300s, inferior ao tempo das simulações deste trabalho, 1000s. A Figura 2 sumariza todos os cenários investigados. Como exemplo de aplicação, um cenário de simulação de 250 veículos, em uma área de $8 \mathrm{~km}$ x $8 \mathrm{~km}$ é destacado.

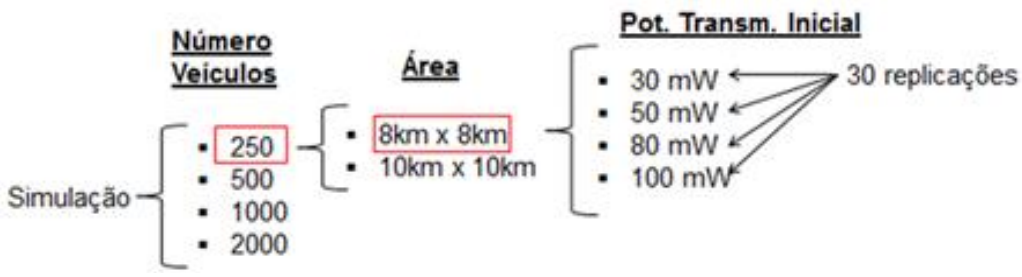

Figura 2: Cenários de Investigação.

\section{Avaliação de desempenho e Discussão dos Resultados}

\subsection{Avaliação das Potências de Transmissão Médias}

Neste tópico são analisados os resultados de potência de transmissão média. Foram simulados quatro cenários: (i) 250, (ii) 500, (iii) 1000 e (iv) 2000 veículos. Para cada um dos cenários acima, foram variados os seguintes elementos: potência de transmissão inicial entre 30 e $100 \mathrm{~mW}$ e sua área de interesse, entre $80 \times 80$ quadras e 100x100 quadras. Por questão de simplicidade, as áreas de simulação de 80x80 quadras são representadas por áreas 80 e as áreas de interesse de 100x100 quadras são representadas por áreas 100.

Como pode ser observado na Tabela 1, a potência de transmissão média dos cenários de área de interesse de 80x80 quadras apresentam valores de potência de transmissão média inferiores que dos cenários de área de interesse de 100x100 quadras. Isto mostra que o algoritmo reduz de forma um pouco maior a potência de transmissão em áreas onde a concentração de veículos é maior. 
Tabela 1: Resultados da Potência de Transmissão para simulação com 250 veículos para potências iniciais de: (i) $30 \mathrm{~mW}$, (ii) $50 \mathrm{~mW}$, (iii) $80 \mathrm{~mW}$ e (iv) $100 \mathrm{~mW}$.

\begin{tabular}{|c|c|c|c|c|c|}
\hline $\begin{array}{c}\text { Potência } \\
\text { Inicial (mW) }\end{array}$ & $\begin{array}{c}\text { Área de } \\
\text { Interesse }\end{array}$ & $\begin{array}{l}\text { Potência de } \\
\text { Transmissão } \\
\text { Média (mW) }\end{array}$ & $\begin{array}{c}\text { Redução da } \\
\text { Potência de } \\
\text { Transmissão (\%) }\end{array}$ & $\begin{array}{c}\text { Intervalo de } \\
\text { Confiança } \\
\text { Inferior } \\
(\mathrm{mW}) \\
\end{array}$ & $\begin{array}{c}\text { Intervalo de } \\
\text { Confiança } \\
\text { Superior } \\
(\mathrm{mW})\end{array}$ \\
\hline 30 & 80 & 29,53 & 1,58 & 29,49 & 29,56 \\
\hline 30 & 100 & 29,68 & 1,07 & 29,64 & 29,72 \\
\hline 50 & 80 & 49,52 & 0,95 & 49,48 & 49,56 \\
\hline 50 & 100 & 49,70 & 0,59 & 49,67 & 49,74 \\
\hline 80 & 80 & 79,46 & 0,68 & 79,42 & 79,49 \\
\hline 80 & 100 & 79,69 & 0,39 & 79,66 & 79,72 \\
\hline 100 & 80 & 99,52 & 0,48 & 99,49 & 99,56 \\
\hline 100 & 100 & 99,70 & 0,30 & 99,66 & 99,74 \\
\hline
\end{tabular}

Nas simulações com 250 veículos, a maior redução na potência de transmissão se dá no cenário de potência de transmissão inicial de $30 \mathrm{~mW}$.

Por fim, a Tabela 2 mostra os resultados de potência média dos veículos para os diversos cenários de simulação com 2000 veículos. Como pode ser observado nos resultados obtidos de potência de transmissão, as maiores reduções de potência de transmissão se dão para a potência de transmissão inicial de $30 \mathrm{~mW}$. Como era de se esperar, a maior redução de potências de transmissão se dá para $30 \mathrm{~mW}$ de potência de transmissão inicial e no cenário de 2000 veículos simulados em um grid de 80x80 quadras.

Tabela 2: Resultados da Potência de Transmissão para simulação com 2000 veículos para potências iniciais de: (i) $30 \mathrm{~mW}$, (ii) $50 \mathrm{~mW}$, (iii) $80 \mathrm{~mW}$ e (iv) $100 \mathrm{~mW}$.

\begin{tabular}{|c|c|c|c|c|c|}
\hline $\begin{array}{c}\text { Potência } \\
\text { Inicial } \\
\text { (mW) }\end{array}$ & $\begin{array}{c}\text { Área de } \\
\text { Interesse }\end{array}$ & $\begin{array}{c}\text { Potência de } \\
\text { Transmissão } \\
\text { Média (mW) }\end{array}$ & $\begin{array}{c}\text { Redução da Potência } \\
\text { de Transmissão (\%) }\end{array}$ & $\begin{array}{c}\text { Intervalo de } \\
\text { Confiança } \\
\text { Inferior (mW) }\end{array}$ & $\begin{array}{c}\text { Intervalo de } \\
\text { Confiança } \\
\text { Superior (mW) }\end{array}$ \\
\hline 30 & 80 & 22,70 & 24,32 & 22,69 & 22,72 \\
\hline 30 & 100 & 24,41 & 18,63 & 24,39 & 24,43 \\
\hline 50 & 80 & 41,15 & 17,69 & 41,13 & 41,18 \\
\hline 50 & 100 & 43,86 & 12,28 & 43,84 & 43,88 \\
\hline 80 & 80 & 70,84 & 11,45 & 70,81 & 70,87 \\
\hline 80 & 100 & 73,78 & 7,78 & 73,75 & 73,80 \\
\hline 100 & 80 & 90,90 & 9,10 & 90,87 & 90,93 \\
\hline 100 & 100 & 93,84 & 6,16 & 93,82 & 93,87 \\
\hline
\end{tabular}




\subsection{Avaliação dos valores médios de SINR}

Nesta análise, são comparados os valores médios de SINR para os diversos cenários. Na simulação de 250 veículos, é sutil, porém perceptível, a diferença entre os valores de SINR dos cenários de área de interesse de 80x80 quadras e 100x100 quadras. No caso de maior concentração, foi possível notar que os valores de SINR são superiores. Além disso, é possível notar que para mesmos valores de concentração veicular, porém maior potência de transmissão inicial, os valores de SINR se mantêm superiores, à medida que a potência de transmissão é aumentada.

Uma redução na SINR nos cenários onde se tem 100x100 quadras mostrou que os veículos estão afastados a ponto de a SINR ser reduzida. O algoritmo proposto então reduz a potência de transmissão sem reduzir a SINR para este cenário. Ou seja, com uma maior concentração veicular, o que resulta em maior interferência, obtém-se uma redução na potência de transmissão média sem reduzir a SINR médio dos veículos.

Os resultados para a simulação com 500 veículos e potências fixas mostraram que os valores de SINR para o cenário de área de interesse de 100x100 quadras é bem próximo, mostrando que não há uma diferença substancial na redução da potência de transmissão em termos de SINR. Porém, para os cenários de área de interesse de 80x80 quadras houve uma diferença na SINR encontrada. Foram obtidos valores inferiores de SINR nos casos do algoritmo proposto.

Por fim, foi notória a redução da potência de transmissão entre os cenários de potência fixa e controlada pelo algoritmo. O que mostra que apesar de uma redução na potência de transmissão média, que pode chegar a $8 \%$ nestes cenários, não interfere nos resultados de SINR do cenário proposto.

\section{Conclusão e Trabalhos Futuros}

O estudo de VANETs está cada vez mais presente em pesquisas que envolvem desde aplicações para VANETs a soluções para roteamento eficiente, eficiência energética com controle de potência de transmissão, controle de mensagens através de QoS aplicado a VANETs e muitos outros. A teoria dos jogos está presente em vários trabalhos que não só se limitam à economia. São muitos trabalhos em redes de sensores, redes móveis celulares, VANETs, robótica, mobilidade urbana e outros.

Este trabalho propõe um algoritmo que modela matematicamente a escolha da potência de transmissão de nós de uma VANET utilizando teoria dos jogos cooperativos. Como diferencial, esta abordagem leva em consideração os efeitos da mudança de taxa de transmissão em uma VANET em alta concentração veicular, estimulando os nós da rede a reduzir a potência de transmissão. Foram analisados cenários com 250, 500 e 1000 veículos simulados e foi mostrado que em cenários de alta concentração veicular o algoritmo proposto reduz a potência de transmissão sem reduzir os valores médios de SINR.

Os resultados mostram que, em um cenário de alta concentração veicular, há uma redução de potência de transmissão média nos módulos de comunicação que pode chegar a uma média de $24,32 \%$ da potência de transmissão inicial. Em um cenário de veículos elétricos, há todo o interesse em se reduzir qualquer tipo de consumo elétrico, 
aumentando assim a eficiência energética do veículo como um todo, sem perder em qualidade de comunicação entre os veículos pertencentes a uma VANET.

\section{Referências}

CHEN, X. Decentralized Computation Offloading Game for Mobile Cloud Computing. IEEE Transactions on Parallel and Distributed Systems, v. 26, n. 4, 2014.

DOUROS, V. G.; POLYZOS, A. G. C. Review of some fundamental approaches for power control in wireless networks. Computer Communications, 34, n. 13, 2011. $1580-1592$.

FUJITSU. FUJITSU Network BroadOne LTE Femtocell Systems , 2013. Disponivel em: <http://www.fujitsu.com/global/products/network/products/femto/>. Acesso em: 2014.

HA, V. N.; LE, L. B. Distributed Base Station Association and Power Control for Heterogeneous Cellular Networks. IEEE TRANSACTIONS ON VEHICULAR TECHNOLOGY, v. 63, n. 1, p. 282-296, 2014.

HAIDER, F. et al. Spectral-Energy Efficiency Trade-off of Cellular Systems with Mobile Femtocell Deployment. IEEE Transactions on Vehicular Technology, v. PP, n. 99, p. 1-12, 2015.

HAN, Z.; LIU, K. J. R. Noncooperative Power-Control Game and Throughput Game Over Wireless Networks. IEEE TRANSACTIONS ON COMMUNICATIONS, v. 53, n. 10, p. 1625-1629, 2005.

KHAYATIAN, H.; SAADAT, R.; ABOUEI, J. Coalition-Based Approaches for Joint Power Control and Relay Selection in Cooperative Networks. IEEE TRANSACTIONS ON VEHICULAR TECHNOLOGY, v. 62, n. 2, p. 835 - 842, 2013.

RAWAT, D. B.; POPESCU, D. C.; OLARIU, G. Y. A. S. Enhancing VANET Performance by Joint Adaptation of Transmission Power and Contention Window Size. IEEE TRANSACTIONS ON PARALLEL AND DISTRIBUTED SYSTEMS, v. 22, n. 9, p. 1528-1535, 2011.

SMITH, D. B. et al. Multi-Source-Destination Distributed Wireless Networks: ParetoEfficient Dynamic Power Control Game With Rapid Convergence. Vehicular Technology, IEEE Transactions on, v. 63, n. 6, p. 2744 - 2754, 2014.

TINGTING CHEN, L. Z. F. W. S. Z. Stimulating Cooperation in Vehicular Ad Hoc Networks: A Coalitional Game Theoretic Approach. IEEE Transactions on Vehicular Technology, -, v. 60, n. 2, p. 566 - 579, 2011.

WANG, Q.; FAN, P.; LETAIEF, K. B. On the Joint V2I and V2V Scheduling for Cooperative VANETs With Network Coding. IEEE Transactions on Vehicular Technology, v. 61, n. 1, p. 62-73, 2012.

YERRAMALLI, S.; JAIN, R.; AND URBASHI MITRA. Coalitional Games for Transmitter Cooperation in MIMO Multiple Access Channels. IEEE TRANSACTIONS ON SIGNAL PROCESSING, 62, n. 4, 2014. 757-771.

YUYI LI, K. Y. P. C. H. Y. A. H. L. Cooperative Data Dissemination in CellularVANET Heterogeneous Wireless Networks , 2012. 